\title{
El género Planois Signoret, 1864 (Heteroptera: Acanthosomatidae) en la Patagonia Chilena
}

\author{
The genus Planois Signoret, 1864 \\ (Heteroptera: Acanthosomatidae) in the Chilean Patagonia
}

Eduardo I. Faúndez ${ }^{1,2}$ \& Mariom A. Carvajal ${ }^{1,2}$

\section{Resumen}

Se analiza y discute la presencia del género Planois en la Patagonia chilena. Se entregan los primeros registros de Planois smaug para la Región de Aysén, extendiendo su distribución hacia el norte. En adición se describe la genitalia interna femenina de esta especie. Se registra por primera vez para Chile Planois patagonus, en base a ejemplares provenientes de la Región de los Lagos. Se concluye que la espermateca es un carácter útil a nivel genérico en Planois, ya que la morfología de esta es común a las tres especies del género. Se analizan y discuten los registros históricos en la literatura de ambas especies y se entrega un mapa actualizado con la distribución de ambas especies en la Patagonia chilena.

\section{Palabras clave:}

Nuevos registros, Hemiptera, Ditomotarsinae, Ditomotarsini, Planois, Chile.

\footnotetext{
Abstract

The presence of the genus Planois in the Chilean Patagonia is analyzed and discussed. The first records from Aysen Region are given for Planois smaug, extending its distribution towards the north. In addition, the female internal genitalia of this species is described for the first time. Planois patagonus is recorded for the first time in Chile, based on specimens from Los Lagos Region. It is concluded that the spermatheca has a generic value on Planois, because it is shared by all the three species on the genus. The historical records on the literature are analyzed and discussed. An
}

updated map of the distribution of Planois species in Chilean Patagonia is provided.

\section{Key words:}

New records, Hemiptera, Ditomotarsinae, Ditomotarsini, Planois, Chile.

\section{INTRODUCCIÓN}

Planois Signoret, 1864 es un género de Acanthosomatidae actualmente ubicado en la subfamilia Ditomotarsinae y tribu Ditomotarsini; este se caracteriza por su gran tamaño y un primer segmento antenal muy elongado, superando el largo de la cabeza (Faúndez et al. 2014). Planois se distribuye en Chile y Argentina, actualmente cuenta con tres especies (Carvajal et al. 2015); sobre su biología, este género ha sido asociado con bosques de Nothofagus Blume (Nothofagaceae) (Carvajal, 2010). En patagonia chilena hasta el momento se han registrado dos especies, Planois gayi (Spinola, 1852) y Planois smaug Carvajal, Faúndez y Rider, 2015. Sin embargo, estos autores indican que la cita de P. gayi probablemente corresponde a otra especie, lo que sugiere una revisión más detallada de este grupo en la región. Por lo tanto, el objetivo

1 Entomology Department, School of Natural Resource Sciences, North Dakota State University, Fargo, ND, USA. \ed.faundez@gmail.com.

2 Departamento de Zoología Médica, Centro de Estudios en Biodiversidad (CEBCh), Magallanes, 1979, Osorno, Chile. 
de esta contribución es clarificar la presencia del género Planois en la patagonia chilena, junto con entregar nuevos registros y datos adicionales de las especies presentes en la zona.

\section{MATERIALES Y MÉTODOS}

Las fotografías fueron tomadas con una cámara digital adaptada a un microscopio estereoscópico. Para el examen de la genitalia interna los ejemplares fueron tratados en una solución saturada de $\mathrm{KOH}$ a $100^{\circ} \mathrm{C}$ por una hora. En terminología morfológica se sigue la usada en Faúndez y Rider (2014). El mapa fue desarrollado con Panmap ${ }^{\circledR}$ Pangaea. El material examinado corresponde a colectas recientes y material de colección, el que se encuentra depositado en la colección de referencia de heterópteros del Centro de Estudios en Biodiversidad (HRCC).

\section{RESULTADOS}

Planois smaug Carvajal, Faúndez \& Rider 2015 (Figs. 1, 2, 4)

Planois bimaculatus (en parte), China (1962): Puerto Edén, Isla Wellington.

Planois bimaculatus (en parte), Pennington (1920): Tierra del Fuego.

Planois gayi, Faúndez (2007): Isla Wellington.

Esta especie fue descrita de Isla Navarino, Región de Magallanes; siendo conocido como el pentatomoideo de mayor envergadura en alcanzar esas latitudes australes (Faúndez, 2015). A continuación entregamos nuevos registros

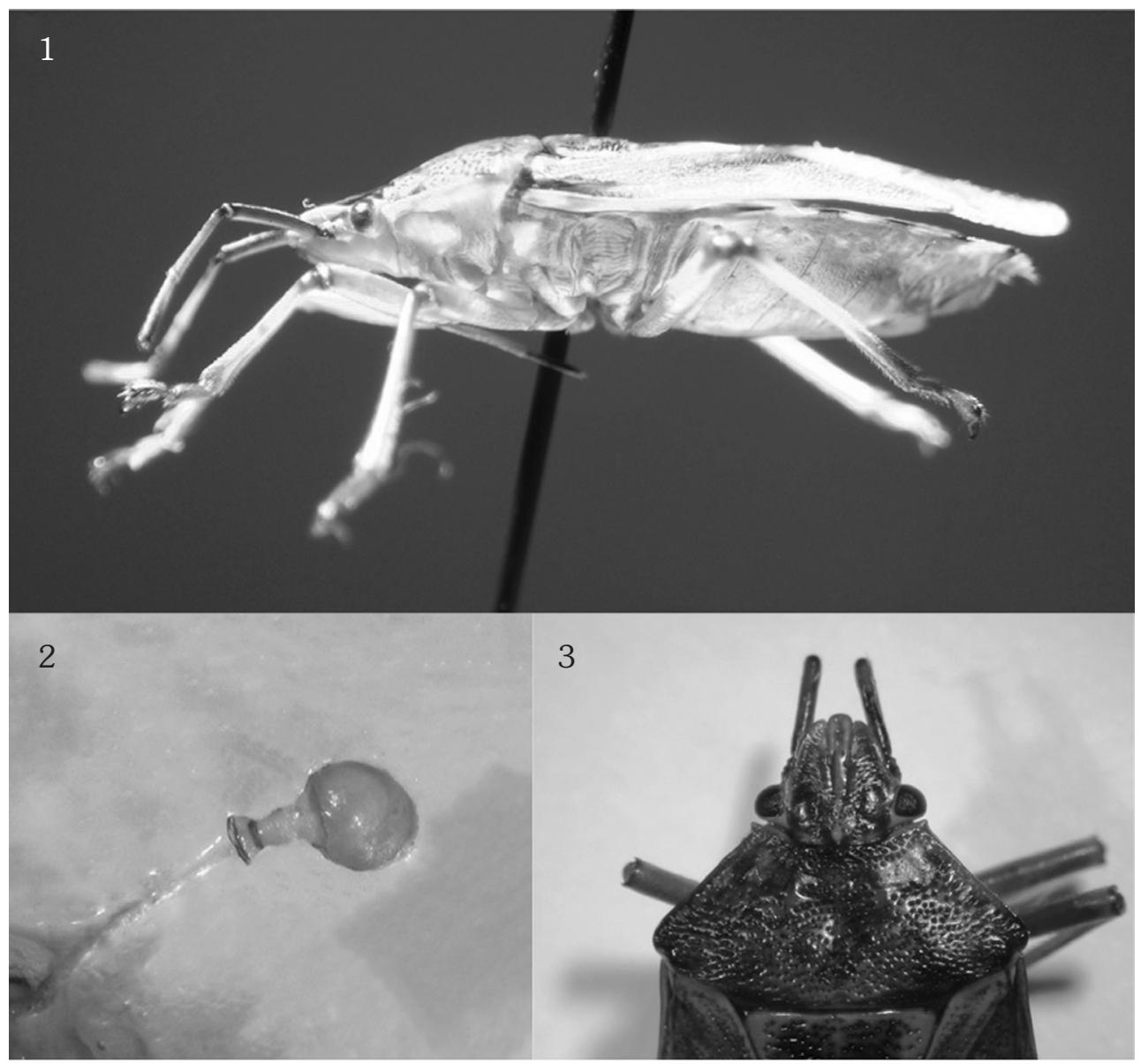

Fig. 1. Planois smaug, vista lateral; Fig. 2. Planois smaug, espermateca; Fig. 3. Planois patagonus, cabeza y pronoto. 


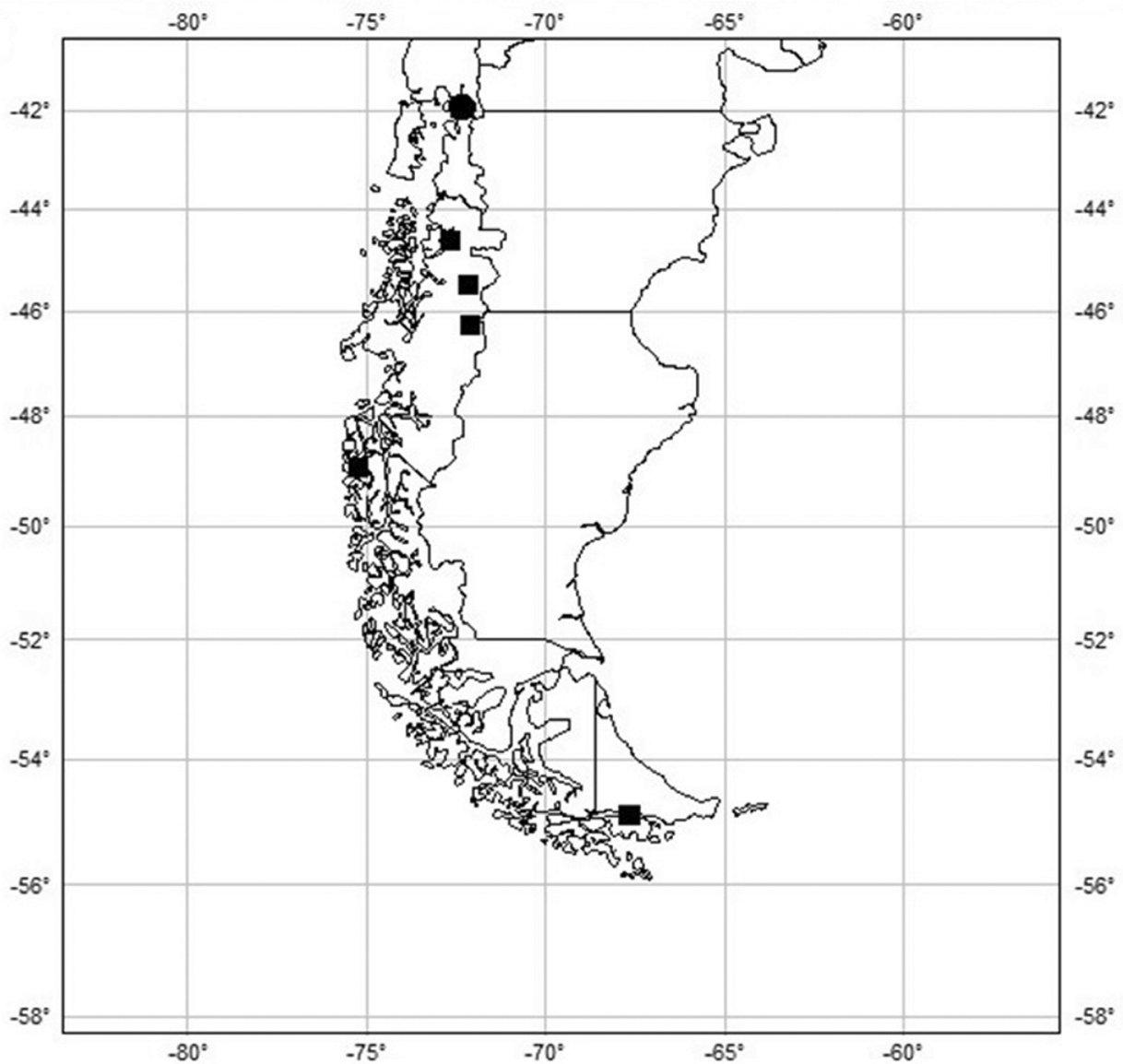

Scale: $1: 17474787$ at Latitude $0^{\circ}$
Fig. 4. Distribución de Planois en Patagonia Chilena, cuadrados $=P$. smaug, circulo $=P$. patagonus. que extienden su distribución hacia el norte y la descripción de la genitalia interna femenina:

Material examinado: CHILE, Región de Aysén, Puerto Cisnes, II-1961, L. E. Peña leg. 1ð̂; Región de Aysén, Coihaique, 8/15-II-2011, A. Lüer leg., $1{ }^{\Uparrow} 1$ q ; Región de Aysén, General Carrera, 8-II2014, A. Lüer leg. 1 ㅇ․

Descripción: Bulbo de la espermateca redondeado (Fig. 2), de apariencia inflada. Reborde proximal del mismo largo que el bulbo, dividido en dos areas morfologicamente distintas (Fig. 2). Área proximal, alargada, en forma de copa, mas delgada posteriormente. Área distal de forma subcónica, trapezoide en vista lateral, con los bordes superiores $e$ inferiores mas oscuros y esclerotizados que el resto de la estructura. Ducto de la espermateca tubular, alargado, de grosor irregular.
Planois patagonus Distant, 1911 (Figs. 3, 4)

Planois patagonus, Carvajal et al. (2015): Patagonia argentina.

Esta especie ha sido citada únicamente de Patagonia argentina, a continuación entregamos el primer registro para Chile:

Material examinado: Chile, Región de Los Lagos, Hornopirén, 26-I-2015, A. Lüer leg., $2 \widehat{\jmath} 3$ 3우.

\section{DISCUSIÓN Y CONCLUSIONES}

El género Planois esta representado por dos especies en la patagonia chilena, Planois smaug y Planois patagonus. La primera se distribuye desde la Región de Aysén hasta la de Magallanes, alcanzando Tierra del Fuego Argentina. La 
espermateca de Planois smaug coincide enormemente con la de $P$. gayi y $P$. patagonus, lo que indicaría que esta estructura es un buen carácter a nivel genérico para Planois, tal como sucede en otros géneros de esta familia (Carvajal et al. 2014). Los nuevos registros permiten confirmar la hipótesis propuesta por Carvajal et al. (2015), que indican que probablemente $P$. smaug se distribuía hacia el norte por la zona de los canales; siendo la única especie presente en el extremo austral de Patagonia Chilena. Las citas de Planois bimaculatus Signoret, 1864 (sinónimo menor de P. gayi) entregadas por China (1962), Pennington (1920) y de P. gayi por Faúndez (2007), en parte corresponden a esta especie. Con la adición de nuevos ejemplares ha sido posible examinar la genitalia de $P$. smaug para confirmar la presencia de esta especie en la zona.

La presencia de Planois patagonus en Chile no es de extrañar, especialmente en esta zona (i.e. cercano a la frontera chileno-argentina en el sur); ya que recientemente se ha descubierto que distintas especies de heterópteros que se creían endémicos de un país u otro, son compartidos por ambos en esta área (e.g. Cornelis et al. 2016; Faúndez \& Carvajal, 2016; Faúndez \& Lüer, 2015; Faúndez \& Rider, 2016); adicionalmente, hemos examinado ejemplares de $P$. patagonus provenientes de El Bolsón, provincia de Río Negro (Argentina), localidad cercana a Hornopirén (Chile). Los ejemplares chilenos concuerdan con los argentinos tanto en genitalia de hembra y macho, puntuación dorsal y lados del pronoto notablemente inclinados que son típicos de esta especie (Carvajal et al. 2015) (Fig. 3).

Aunque no se conoce con exactitud una planta hospedadora para las especies de Planois presentes en patagonia, tanto $P$. smaug como $P$. patagonus, han sido colectadas en bosques de Nothofagus; siendo hasta ahora los únicos datos biológicos conocidos para ambas especies. Por lo tanto, es necesario mejorar los esfuerzos de colecta y prospección para dilucidar los diferentes aspectos de la biología de ambas especies en la zona.

\section{AGRADECIMIENTOS}

Agradecemos a Alfredo Lüer por ceder ejemplares de Planois utilizados en este trabajo.

\section{LITERATURA CITADA}

Carvajal, M. A. (2010). Nuevos registros para Planois gayi (Spinola, 1852) (Hemiptera: Acanthosomatidae) en la Región de la Araucanía, Chile. Boletín de la Sociedad Entomológica Aragonesa, 49, 357-358

Carvajal, M. A., Rider, D. A., \& Faúndez, E. I. (2014). Preliminary studies on the spermatheca of the Acanthosmatidae (Hemiptera: Heteroptera). Proceedings of the quadrennial meeting of the International Heteropterists Society, 5, 24.

Carvajal, M. A., Faúndez, E. I., \& Rider, D. A. (2015). Contribución al conocimiento de los Acanthosomatidae (Hemiptera: Heteroptera) de la Región de Magallanes, con descripción de una nueva especie. Anales del Instituto de la Patagonia, 43(1), 145-151.

China, W. E. (1962). Hemiptera-Heteroptera collected by the Royal Society expedition to South Chile 1958-1959. Annals and Magazine of Natural History, 13(5), 705723.

Cornelis, M., Diez, F. \& Coscaron, M. C. (2016). Description of nymphs and additional information on Nabis ashworthi (Hemiptera: Heteroptera: Nabidae) from Patagonia, Argentina. Acta Entomologica Musei Nationalis Pragae, 56(1), 61-70.

Faúndez, E. I. (2007). Lista de las especies de Acanthosomatidae (Insecta: Hemiptera) de la región de Magallanes (Chile). Anales del Instituto de la Patagonia, 35(1), 75-78.

Faúndez, E. I. (2015). Patagonian Shield Bug Named After Middle's Earth's Smaug the Dragon. Entomology Today (Entomological Society of America), disponible en linea: https:// entomologytoday.org/2015/06/19/ patagonian-shield-bug-named-after-middlesearths-smaug-the-dragon/ [27-7-2016].

Faúndez, E. I., \& Carvajal, M. A. (2016). Description of the 5th instar nymph of Oenopiella punctaria (Stål, 1859) (Hemiptera: Heteroptera: Pentatomidae), with new distributional records from Southern Patagonia. Zootaxa, 4067(2), 246-250.

Faúndez, E. I., \& Lüer, A. (2015). First record 
of Stenocader mapu Carpintero \& Montemayor, 2008 (Heteroptera: Tingidae) from Chile. Anales del Instituto de la Patagonia, 43(1), 163-164.

Faúndez, E. I. ,\& Rider, D. A. (2014). Thestral incognitus, a new genus and new species of Pentatomidae from Chile (Heteroptera: Pentatomidae: Pentatominae: Carpocorini). Zootaxa, 3884(4), 394-400.

Faúndez, E. I., \& Rider, D. A. (2016). Notas sobre el complejo de Acledra (Acledra) albocostata
(Spinola, 1852) (Hemiptera: Heteroptera: Pentatomidae: Pentatominae). Arquivos Entomolóxicos, 15, 383-389.

Faúndez, E. I., Carvajal, M. A., \& Rider, D. A. (2014). Archaeoditomotarsus crassitylus, gen. and sp. nov. from Chile. Zootaxa, 3860(1), 87-91.

Pennington, M .S. (1920). Lista de los Hemipteros Heteropteros de la República Argentina. Primera parte. Pentamoidea- Coroidea, pp. 1-16, Buenos Aires. 
